\title{
A personal view on reviewing the psychological consequences of predictive genetic testing for late onset disease
}

The recent review of Broadstock et $a l^{1}$ is a very ambitious undertaking. It is a challenge to make a critical and genuine synthesis of the relevant findings from the many quantitative and qualitative studies that have been published regarding the complex topic of psychological consequences of predictive testing for late onset disease. Moreover it is difficult to make accurate and meaningful comparisons across diverse neurogenetic diseases and hereditary cancers. This may explain why the very promising title raises expectations that are not fulfilled by the 'systematic' review.

In my opinion the authors should have referred to other important publications ${ }^{2-6}$ about predictive testing and its psychological consequences in order to put things in an adequate perspective. Their introduction of less than 20 lines is mainly limited to the aim of their systematic review: 'to summarise published, empirical data describing the emotional, cognitive and behavioural consequences of undergoing predictive testing'. Unfortunately they do not even discuss what they consider as possible outcome measures for the three dimensions and why.

Moreover when a systematic review neglects qualitative data, it can only result in a 'narrow and superficial view' on the consequences of predictive testing. It is hard to understand why the authors have deliberately neglected the multifaceted experiences and findings of many researchers over the world who have so far described and/or analysed the psychological consequences of predictive testing. The adjective 'systematic' should not be used as an excuse to set aside qualitative studies and empiric studies that do not use the same (simple) standardised measures in the pretest and the post test period. Of course other data are less easy to convert in large overview tables.

Besides being only systematic in a narrow sense, the review is incomplete and partially incorrect. The methods section starts with a description of the search strategy. Here a first problem arises: in a review on predictive genetic testing, it is hard to understand why the terms 'predictive testing' or 'asymptomatic/presymptomatic diagnosis' were not used right from the start. This fact increased the risk of missing relevant papers on predictive testing (some were missed), but it was probably also at the origin of another problem. Indeed (at least) four of the 15 selected papers (their references 12-14 about HBOC and reference 16 about SCA) do not only include persons who had a predictive test, but also affected persons who had a diagnostic genetic test (quite a different situation). In the corresponding rows of Table 2 this leads to 'strange' combinations; this is most obvious for paper 13. The impossibility to distinguish asymptomatic persons from affected ones, so that some data could not be used, is problematic. It is surprising that this important shortcoming is only mentioned in a succinct and unclear way in the discussion, after the mixed data have been used in some tables throughout the paper. Another problem is that the narrow selection criteria result in a limited number of late onset diseases in this review and the dominant presence of Huntington's disease: eight papers were exclusively about HD, one paper about SCA (reference 16 with predictive and diagnostic tests), the six others about hereditary cancers: one about FAP in children, one about FAP in adults and four about HBOC (one HBOC as compared to HD and the three others including both predictive and diagnostic tests, $c f$. supra). This is why the title of the systematic review is too broad and more promising than the content. Moreover the fifth search strategy mentioned in the methods section (the citations of two groups 'producing early relevant papers') is inadequate, because the two groups mentioned represent only a subset of the 'pioneers'.

Reading the results section requires a lot of effort, mainly because of the way the tables are composed-usually frameworks without visible (vivid) content-but also because of a lack of information regarding the real meaning of the headings of the columns (a clear explanation in the text is also lacking). The latter is particularly true for Table 2 . Moreover-based on a check starting from data of our own unit-it is clear that several tables are incomplete and/or include errors. It would be too time consuming for a reader to do a similar check for the other studies, but it is hard to believe that the problem in reporting our data is the only exception and that all other data are completely and correctly reported.

The parts of the discussion that are directly linked to the systematic review show that it is hard to draw general conclusions. This is not surprising: only one of the reviewed studies had a follow-up period of 3 years and more than half of the others had a follow-up period of less than 1 year (one study had a period of only 1-2 weeks but was nevertheless selected for this review). Moreover, because studies on predictive testing for other diseases are scarce or even absent in this review (HNPCC, MEN 2, ALS, von Hippel Lindau) generalisations are definitely not allowed. In my opinion the authors' suggestion 'that the treatability of the condition is 
not a key factor in determining emotional responses' (based on the fact that this systematic review detected no differences in the pattern of results across the conditions HD and HBOC) should be approached with caution, because part of the HBOC data seems problematic ( $c f$. supra) and because the sample size in some studies is so small.

Some parts of the discussion that are not directly linked to the systematic review are more interesting, but are a repetition of suggestions, considerations and statements that can be found in other publications, mainly but not exclusively about HD. As anyone in the field knows, several centres in the world have set up prospective longitudinal studies that are still continuing. Some of them do not publish all their intermediate results on small samples but prefer to end up with a more important message on the long term psychological impact of predictive testing. Therefore the authors' suggestion 'to set up prospective studies over longer time' is a rather superfluous suggestion, because this process has already been going on many years for neurogenetic diseases and in more recent years also for hereditary cancers.

The authors claim that 'this review suggests that testing protocols should include pre-test assessment of emotional state so that post-test counselling can be targeted at those more distressed before testing'. This can certainly not be considered as a new suggestion based on this review. Indeed it was already mentioned in less explicit terms in the Guidelines for predictive testing for $\mathrm{HD}^{7}$ elaborated before the availability of the predictive test as a clinical service and revised after the identification of the gene. Moreover the same statement has been made in several publications about HD during the last decade (some of them are present in the reference list of the review paper, some are not; some are included in the selected papers for the systematic review, some are not).

In conclusion: due to the type of selection process and the neglect of many interesting data published over the years (and limited involvement in interesting European and international meetings paying particular attention to the topic of predictive testing?), the paper by Broadstock et al ${ }^{1}$ is rather disappointing. In addition I am very concerned about the authors' conviction that 'there is a pressing need for experimental studies' manipulating the amount or type of counseling provided. Ethical considerations may be an impediment for these types of studies. This point also merits an exchange of views with the patient associations for several late onset diseases. Those who are regularly in touch with predictive test applicants and who have more contact with the work of health professionals involved in pretest and posttest counseling, would be very reluctant to suggest these types of experimental studies. I also wonder what the added value can be of 'large' international studies using (simple) standardised measures as the authors suggest. For the international dimension and comparison I would personally favour the promising collaborative work that is already going on in smaller European and international collaborations. In this type of collaboration it is possible to pay more attention to qualitative data and to be less dependent on the use of simple statistics (eg means), that mask important interindividual differences or the existence of specific subgroups.

Prof Gerry Evers-Kiebooms Psychosocial Genetics Unit, Center for Human Genetics, 3000 Leuven, Belgium

\section{References}

1 Broadstock M, Michie S, Marteau T: Psychological consequences of predictive genetic testing. Eur J Hum Genet 2000; 8: 731 - 738.

2 Kessler S: Predictive testing for Huntington's disease: A psychologist's view. Am J Med Genet 1994; 4: 161-166.

3 Harper P: Huntington's disease. Saunders, London, 1996.

4 Harper P, Clarke A: Genetics, Society and Clinical Practice. Bios, Oxford, 1997.

5 Evers-Kiebooms G, Decruyenaere M: Predictive testing for Huntington's disease: A challenge for persons at risk and for professionals. Patient Educ Counsel 1998; 35: 15-26.

6 Evers-Kiebooms G, Welkenhuysen M, Claes E, Decruyenaere M, Denayer L: The psychological complexity of predictive testing for late onset neurogenetic diseases and hereditary cancers: Implications for multidisciplinary counselling and for genetic education. Soc Sci Med 2000; 51: 831-841. (This paper was already available-as discussion paper on their website-since September 1999).

7 International Huntington Association and World Federation of Neurology: Guidelines for the molecular genetics predictive test in Huntington's disease. Neurology 1994; 44: 1533-1536.

\section{Reply to letter from G Evers-Kiebooms}

The letter from Evers-Kiebooms responding to our systematic review of the psychological consequences of predictive genetic testing ${ }^{1}$ raises four main points.

(1) Qualitative data should be included in systematic reviews.

(2) Other research is consistent with the findings of the systematic review.

(3) It is unethical to carry out experimental studies (randomised controlled trials) of genetic counselling.

(4) The review includes some unspecified inaccurate reporting.

We will discuss these points in the same order:

(1) Systematic reviews vary in their inclusion criteria, depending on the research question they are addressing. In our review, we were interested in the outcome of testing as assessed by standardised, quantitative measures of psychological functioning. We chose to do this in order to enable comparisons across studies that vary across several dimensions. Systematic reviews are just that: systematic in the way that they define and pursue their search and integration of the relevant literature. ${ }^{2}$ Our search strategy used terms that maximised the chance of identifying the studies of interest, and this was confirmed by our validation check. 
Systematic reviews can only be as good as the research that they review and we described some of these limitations, such as a lack of cognitive and behavioural outcomes, and study groups which combined affected with unaffected people (which were therefore omitted from our review). Qualitative studies have an important contribution to make to understanding psychological impact, and a review of this work would be very timely. The large number of reprint requests for this systematic review suggests a great interest in this area, and our review is only one, and certainly not definitive, contribution.

(2) Other studies of predictive genetic testing exist that did not meet our review criteria have also found that pre-test mood is an important determinant of post-test mood. This does not detract from the importance of any of those findings. Indeed, the more consistency between different studies carried out in different countries on different conditions using different methods, the more robust that finding is.

(3) As evidence-based health care is increasingly advocated as the most ethical approach, it becomes necessary to define what constitutes evidence. The strongest evidence about causation (eg, that a type of counselling causes a type of outcome) comes from studies that compare models of care in which only the aspects of interest are varied, whilst keeping others the same. This experimental approach is further strengthened by randomising people to these two conditions so that we can be confident that it is the type of counselling that is bringing about the outcome, not the type of person who selects the type of counselling. To deprive people of the evidence on which to base their health care choices is ethically problematic.

The studies we envisage would not deprive anyone of counselling. Rather, they might compare different amounts and types of counselling at different stages of the testing process to determine the most effective and efficient counselling for different groups of counsellees. Current practice of predictive testing counselling varies widely. Without evidence to support any one type of practice, those commissioning care will understandably select the cheapest, which may not be the best, option. It is arguably unethical not to conduct experimental studies that address this issue.

(4) Whilst we acknowledge that any published research may include inaccuracies, our review was checked by three researchers and we have received no details about inaccuracies within it.

We hope that our review, and this subsequent correspondence, will help to stimulate interest in, and study of, this area. Further research, building on what we know to date, will help to further our understanding of the psychological and social consequences of predictive genetic testing and how to present such testing in a way that maximises well-being and minimises harm.

$$
\begin{array}{r}
\text { Susan Michie, Theresa Marteau } \\
\text { Psychology and Genetics Research Group, } \\
\text { Guy's, King's and St Thomas' Medical School, } \\
\text { London, UK }
\end{array}
$$

\section{References}

1 Broadstock M, Michie S, Marteau TM: The psychological consequences of predictive genetic testing: a systematic review. Eur J Hum Genet 2000; 8: 731 - 738 .

2 Mulrow CD: Rationale for systematic reviews. Br Med J 1994; 309: $597-599$. 